\title{
Shrinkages in heavy-sized cast components of nodular cast iron - NDT and fatigue
}

\author{
Christoph Bleicher, Heinz Kaufmann and Rainer Wagener
}

\begin{abstract}
Material defects like shrinkages, dross, pores and chunky graphite are likely to occur in thick-walled castings and are a challenge for the foundries and their customers. These defects are mostly detected with handheld ultrasonic testing (UT) or X-ray analysis. Within a research project done at the Fraunhofer Institute for Structural Durability and System Reliability LBF, the fatigue of Dross, shrinkages and chunky graphite in thickwalled cast material GGG-40 was estimated based on X-ray and fatigue tests on bending specimens. High fatigue reductions were received for the different material imperfections. Based on these impressions a further research project was executed at the Fraunhofer LBF to get an estimation of the informational value of UT in relation to fatigue of shrinkages in thick-walled castings of the material EN-GJS-400-18U-LT, EN-GJS-450-18 and ENGJS-700-2. With the help of X-ray analysis and the UT technique Sampling Phased Array (SPA) information about geometry and density were derived for a numerical analysis of shrinkages in thick-walled castings concerning fatigue. The following text summarizes the fatigue results achieved in the two research projects with the help of the X-ray and UT analysis.
\end{abstract}

For thick-walled components of nodular cast iron material defects like shrinkages, dross, pores and chunky graphite are likely to occur and are a challenge for the foundries and their customers. These defects are mostly detected with handheld ultrasonic testing (UT) or X-ray analysis, whereas the last method is not appropriate to be applied with every possible component size and will not be performed within a serial production in a foundry. Although ultrasonic testing is a common method to test critical parts of heavy-sized cast components, a detailed decision whether the defect found is really adverse for the component, is not possible. Also there will be no information about the real size, depth or density of the imperfection given by the UT which will help to recalculate the affected position in the component by FEM correctly. With non-destructive testing (NDT) using UT foundries classify their products according to the quality classes in order to certify the specific cast component. Although this is a common way, the identification of a cast quality over the quality classes is not a proper method to identify the component's real fatigue behaviour. Additionally the quality classes give no information about the position of the e.g. shrinkages related to the highly stressed volume.

In a research project (AiF-project No. 9155) finished at Fraunhofer LBF in 1998 the material imperfections dross, shrinkages and chunky-graphite in thick-walled castings of GGG-40 were investigated using all-side machined bending specimens with a stress concentration factor of $\mathrm{K}_{\mathrm{t}}=1.10$ by testing them until $\mathrm{N}_{\mathrm{lim}}=1 \cdot 10^{7}$ cycles. Sound and defect specimens were taken from especially 


\section{MATEC Web of Conferences}

casted blocks with $120 \mathrm{~mm}$ thickness and from deficient cast products. The sound material was used to determine the material's properties. For a probability of survival $\mathrm{P}_{S}=50 \%$ under alternating loading, $\mathrm{R}_{\sigma}=-1$, a stress amplitude of $\sigma_{\mathrm{a}, \mathrm{k}}=141 \mathrm{MPa}$ was received at the Wöhler curve's knee-point of $\mathrm{N}_{k}=2 \cdot 10^{6}$ cycles with a mean stress sensitivity of $\mathrm{M}=0.29$.

For specimens containing chunky-graphite the nominal stress amplitude is lowered to an amplitude of $\sigma_{\mathrm{a}, \mathrm{k}}=111 \mathrm{MPa}$ at a knee-point of $\mathrm{N}_{k}=2 \cdot 10^{6}$ cycles under alternating loading. The investigated dross formation led to a decrease of the stress amplitude down to $\sigma_{\mathrm{a}, \mathrm{k}}=75 \mathrm{MPa}$. The mean stress sensitivities for the investigated chunky-graphite and dross were much higher than the sound material and reached $\mathrm{M}=0.48$.

For the investigations of shrinkages all bending specimens were X-ray analysed and the shrinkage classes according the ASTM-E802 norm were evaluated. For each of the defined classes Wöhler curves were determined. For the highest shrinkage class only a stress amplitude of $\sigma_{\mathrm{a}, \mathrm{k}}=74 \mathrm{MPa}$ could be found for the stress ratio $\mathrm{R}_{\sigma}=-1$ and $\mathrm{N}_{k}=2 \cdot 10^{6}$ cycles.

Based on these former investigations, the Fraunhofer institutes LBF and IZFP (Institute for Nondestructive Testing) worked together with partners from foundries and their purchasers in order to combine new methods of UT with the fatigue behaviour of shrinkages in nodular cast iron (EN-GJS400-18U-LT, EN-GJS-450-18 and EN-GJS-700-2) in heavy-sized components mainly used for the wind energy division. Besides a further development of mechanised UT and its technology Sampling Phased Array (SPA), the fatigue behaviour of axial fatigue specimens with shrinkages was investigated with stress controlled tests. Test specimens were taken from cast blocks ( $120 \mathrm{~mm}$ up to $230 \mathrm{~mm}$ in thickness) and components provided by the project partners.

All test blocks and components were tested with handheld, mechanised UT and SPA. The SPA technology specifies a defect with a much higher precision and enables the user to derive 3D geometry information of the defect inside the material. With detailed information of the position and the geometry of the shrinkages inside the blocks and components sound and defect axial fatigue specimens could be extracted quite precisely and with a high traceability. More than 110 specimens with shrinkages of the three materials were taken from the castings and tested under cyclic axial loading. For the purpose of reference again all withdrawn specimens were 2D-X-ray as well as CT-X-ray (3D) analysed and the 2DX-ray results were classified according to ASTM-E446. The CT analysis derived knowledge about size, distribution and form of the shrinkages and the density of the specimens' test volume. The calculated density in the test area of the specimens, based on the CT-X-ray analysis of the shrinkages, was verified by weighing the specimens after the fatigue tests.

For each of the three materials and shrinkage classifications Wöhler curves at a stress ratio of $\mathrm{R}_{\sigma}=-1$ were derived. As a reference sound specimens were taken from the cast blocks close to the shrinkages and tested amongst others under alternating loading up to $\mathrm{N}_{\lim }=1 \cdot 10^{7}$ cycles. The three tested materials reached stress amplitudes of $\sigma_{\mathrm{a}, \mathrm{lim}}=137 \mathrm{MPa}$ for the EN-GJS-400-18U-LT, $\sigma_{\mathrm{a}, \mathrm{lim}}=153 \mathrm{MPa}$ for the solid-solution strengthened EN-GJS-450-18 and $\sigma_{\mathrm{a}, \text { lim }}=175 \mathrm{MPa}$ for the EN-GJS-700-2 respectively for $\mathrm{R}_{\sigma}=-1$ at $\mathrm{N}_{\lim }=1 \cdot 10^{7}$ cycles and $\mathrm{P}_{\mathrm{S}}=50 \%$. For the mean stress sensitivities quite high values between $\mathrm{M}=0.46$ and $\mathrm{M}=0.56$ were derived.

Independent of the fatigue strength of the sound specimens the shrinkage afflicted specimens of all investigated materials reached similar values for the high cycle fatigue regime with a rising ASTM classification. This effect is related to the notch effect of the shrinkages. Hence, a common evaluation of the fatigue results of all materials within one shrinkage class makes sense and based on this the nominal stress amplitudes for each classification could be set up. For the highest shrinkage classification Cc-5, a nominal stress amplitude of $\sigma_{\mathrm{a}, \mathrm{lim}}=62 \mathrm{MPa}$ at $\mathrm{N}_{\mathrm{lim}}=1 \cdot 10^{7}$ cycles was obtained. For the fatigue test based approach of shrinkages in the three axially tested materials a maximum scatter band of $\mathrm{T}_{\sigma}=1: 1,80$ was found.

The tests of the axial specimens with shrinkages showed quite similar results for the nominal stress amplitudes at $1 \cdot 10^{7}$ cycles to the ones derived for the GGG-40. But the scatter band increased 
considerably from $\mathrm{T}_{\sigma}=1: 1,20$ for the GGG-40 found in the first project under bending load to $\mathrm{T}_{\sigma}=1: 1,80$ for the shrinkage related Wöhler curves of the combined evaluation of the three materials in the second project.

Due to this high value, further research needs to be done to reduce the scatter band with reasonable and reproducible methods. 